\title{
Absence of hyperuniformity in amorphous hard-sphere packings of nonvanishing complexity
}

\author{
M. J. Godfrey and M. A. Moore \\ School of Physics and Astronomy, University of Manchester, Manchester M13 9PL, UK
}

(Dated: August 21, 2018)

\begin{abstract}
We relate the structure factor $S(\mathbf{k} \rightarrow \mathbf{0})$ in a system of jammed hard spheres of number density $\rho$ to its complexity per particle $\Sigma(\rho)$ by the formula $S(\mathbf{k} \rightarrow \mathbf{0})=-1 /\left[\rho^{2} \Sigma^{\prime \prime}(\rho)+2 \rho \Sigma^{\prime}(\rho)\right]$. We have verified this formula for the case of jammed disks in a narrow channel, for which it is possible to find $\Sigma(\rho)$ and $S(\mathbf{k})$ analytically. Hyperuniformity, which is the vanishing of $S(\mathbf{k} \rightarrow \mathbf{0})$, will therefore not occur if the complexity is nonzero. An example is given of a jammed state of hard disks in a narrow channel which is hyperuniform when generated by dynamical rules that produce a non-extensive complexity.
\end{abstract}

Systems that are hyperuniform have small long-wavelength density fluctuations. In other words, their structure factor $S(\mathbf{k})$ vanishes as the wavevector $\mathbf{k} \rightarrow \mathbf{0}$. The structure factor of $N$ spheres whose centers are at $\mathbf{r}_{j}$ is defined as $S(\mathbf{k})=\left|\rho_{\mathbf{k}}\right|^{2} / N$, where the Fourier transform of the number density $\rho_{\mathbf{k}}=\sum_{j=1}^{N} \exp \left(-i \mathbf{k} \cdot \mathbf{r}_{j}\right)$. The whole subject of hyperuniformity has been recently reviewed at length by Torquato [1]. In this paper we focus on one of the most extensively studied questions connected with hyperuniformity the extent to which jammed systems of hard spheres or disks are hyperuniform. Torquato and Stillinger [2] have made the following conjecture:

Any strictly jammed saturated infinite packing of identical spheres in $\mathbb{R}^{d}$ is hyperuniform.

The restrictions on the packing are important. A "strictly" jammed packing is one such that each particle is firstly "locally" trapped by its neighbors so that it cannot be translated while their positions are held fixed and secondly is also "collectively jammed" so that no subset of the particles can be simultaneously moved so that its members move out of contact with one another with the remainder set fixed. Finally, the condition of strict jamming precludes any uniform volume-decreasing strains of the boundary, which means that their bulk and shear moduli are infinitely large [1]. The word "saturated" in the conjecture eliminates packings that contain voids large enough to accommodate a "rattler" [1]. Such voids would be a source of diffuse scattering, leading to a nonzero limiting value for the structure factor, $S(\mathbf{k} \rightarrow \mathbf{0}) \neq 0$.

Many investigators have reported that the limit $S(\mathbf{k} \rightarrow \mathbf{0})$ seems to be non-zero for jammed packings of hard spheres in three dimensions; Refs. [3, 4] are recent examples. In response, Torquato has asserted that the "difficulty of ensuring jamming as $N$ becomes sufficiently large to access the small-wavenumber regime in the structure factor as well as the presence of rattlers that degrade hyperuniformity makes it virtually impossible to test the Torquato-Stillinger jamminghyperuniformity conjecture via current numerical packing protocols" [1].

Ikeda and Berthier [3], who found that $S(\mathbf{k} \rightarrow \mathbf{0}) \neq 0$ in their work, observed that it is "difficult to provide a physical explanation for the existence of the observed deviations from strict hyperuniformity, mainly because there is no deep physical reason to expect perfect hyperuniformity in these systems in the first place". In this paper we argue for the correctness of this view, by showing that at least in the regime where the logarithm of the number of jammed states (a quantity usually referred to as the complexity $\Sigma_{N, V}$ ) is extensive and proportional to $N$ one can derive a simple formula for the finite value of $S(\mathbf{k} \rightarrow \mathbf{0})$ in terms of derivatives of $\Sigma_{N, V}$. Our formula for $S(\mathbf{k} \rightarrow \mathbf{0})$ is the analog of that for an equilibrium system at temperature $T$ :

$$
S(\mathbf{k} \rightarrow \mathbf{0})=\frac{\left\langle M^{2}\right\rangle-\langle M\rangle^{2}}{\langle M\rangle}=\rho k_{B} T \kappa_{T},
$$

where $\rho$ is the number density $N / V$, and $\kappa_{T}$ is the isothermal compressibility [1]; $M$ is the number of particles within a large sub-volume (window) of the system of volume $\Omega$ so $\langle M\rangle=\rho \Omega$. The name "hyperuniformity" means that the variance of the number of particles in $\Omega$ is vanishingly small compared to the natural expectation that it should be proportional to $M$. Hard sphere fluids at a temperature $T$ are not hyperuniform as their compressibility $\kappa_{T}$ is finite.

We shall first re-write Eq. (1) in a more useful form for our discussion of jammed states. Let $p$ be the pressure in the system. The compressibility $\kappa_{T}$ is related to the second derivative of the Helmholtz free energy $F$ of the system, $F=E-T S$, where $E$ is the energy of the system and $S$ is its entropy:

$$
\kappa_{T} \equiv-\frac{1}{V}\left(\frac{\partial V}{\partial p}\right)_{T, N}=\frac{1}{V} \frac{1}{\left(\partial^{2} F / \partial V^{2}\right)_{T, N}} .
$$

For a system of hard spheres in $d$ dimensions, $E=\frac{1}{2} N d k_{B} T$, so that we can write $\left(\partial^{2} F / \partial V^{2}\right)_{T, N}=-T\left(\partial^{2} S / \partial V^{2}\right)_{T, N}$. Then Eq. (1) becomes

$$
S(\mathbf{k} \rightarrow \mathbf{0})=-\frac{\rho}{V} \frac{k_{B}}{\left(\partial^{2} S / \partial V^{2}\right)_{T, N}} .
$$

We turn now to generalizing Eq. (3) to jammed states. We imagine that an averaging process is used. The simplest is the Edwards procedure of giving equal weight to all the jammed states of a given number density $\rho$. Let us call the complexity $\Sigma_{N, V}$, the logarithm of the number of jammed states of $N$ spheres in a volume $V$. Numerical methods have been recently devised for determining $\Sigma_{N, V}$ [5]. When it is extensive, it is expected to be of the form $\Sigma_{N, V}=N \Sigma(\rho)$ as $N \rightarrow \infty$ at fixed density $\rho \equiv N / V$. It is then our claim that 
the average of the structure factor $S(\mathbf{k} \rightarrow \mathbf{0})$ over the jammed states can be obtained from Eq. (3) by making the replacement

$$
S / N \rightarrow k_{B} \Sigma(\rho) .
$$

This kind of idea is clearly related to those that have been successfully used in the theory of granular systems [6]. In terms of derivatives of the function $\Sigma(\rho)$, Eqs. (3) and (4) yield

$$
S(\mathbf{k} \rightarrow \mathbf{0})=-\frac{1}{\rho^{2} \Sigma^{\prime \prime}(\rho)+2 \rho \Sigma^{\prime}(\rho)} .
$$

Hyperuniformity can arise if the function $\Sigma(\rho)$ has divergent first or second derivatives, which is only likely to happen at exceptional values of $\rho$; we shall see later that for a system of disks in a narrow channel this happens at the two packing fractions, $\phi_{\min }$ and $\phi_{\max }$, for which the system has crystalline order. In general, the right hand side of Eq. (5) will not be zero and there will be no hyperuniformity. However, if the complexity is not extensive, Eq. (5) cannot be used and hyperuniformity may then occur. Later we shall give an explicit example of such behavior for a system of hard disks in a narrow channel for which the configuration has been generated by a set of dynamical rules that result in a non-extensive complexity.

The result in Eq. (5), obtained by analogy with the equilibrium theory, can be derived directly for jammed systems. Denote by $V\left[C_{N}\right]$ the volume of a jammed configuration $C_{N}$ of $N$ spheres. Then the complexity $\Sigma_{N, V}$ is given by

$$
e^{\Sigma_{N, V}}=\sum_{C_{N}} \delta\left(V-V\left[C_{N}\right]\right)
$$

where we follow the Edwards prescription [6] of giving equal weight to each of the jammed states. (We discuss other averaging possibilities later.) Often it is easier to work with the Legendre transform of Eq. (6), and discuss

$$
Z_{N, f}=\sum_{C_{N}} e^{-f V\left[C_{N}\right]}=\int e^{\Sigma_{N, V}-f V} d V .
$$

The reciprocal of $f$ is what Edwards and Oakeshott have referred to as the "compactivity" [6].

If $\Sigma_{N, V}$ is extensive, the integral on the right-hand side of Eq. (7) can be done by steepest descents. Stationarity of the argument of the exponential gives

$$
f=\frac{\partial \Sigma_{N, V}}{\partial V}=-\rho^{2} \frac{d \Sigma(\rho)}{d \rho},
$$

where the last expression follows by writing $\Sigma_{N, V}=N \Sigma(\rho)$ with $\rho=N / V$. Equation (8) determines $f$ as a function of the density $\rho$.

We note here that $\Phi(N, f) \equiv-\ln Z_{N, f}=f V-\Sigma_{N, V}$ is the athermal analog of the Gibbs free energy. As in thermodynamics, one may use the extensivity of $\Phi$ to show that $\Phi=N \mu(f)$, where $\mu=-\partial \Sigma_{N, V} / \partial N$ corresponds to the chemical potential.
The structure factor is given by the usual expression $S(\mathbf{k})=\left\langle\tilde{\rho}_{\mathbf{k}} \tilde{\rho}_{-\mathbf{k}}\right\rangle / N$, where $\tilde{\rho}_{\mathbf{k}}$ is the Fourier transform of $\tilde{\rho}(\mathbf{x})=\rho(\mathbf{x})-\langle\rho\rangle$, the deviation of $\rho(\mathbf{x})$ away from its average value. The subtraction removes from $S(\mathbf{k})$ a $\delta$-function at $\mathbf{k}=\mathbf{0}$ and makes it possible to show that

$$
S(\mathbf{k} \rightarrow \mathbf{0})=\frac{\operatorname{var} M}{\langle M\rangle},
$$

where $M$ is the number of particles in a window of volume $\Omega \ll V$, placed at random in $V$, in the limit where $\Omega$ and $\langle M\rangle$ are large [1].

To find the distribution of $M$ we adopt an approach that is similar to the elementary one used to derive the Gibbs distribution in statistical physics. In a typical state drawn from the Edwards ensemble, the number of spheres $M$ whose centers lie within a randomly-placed window of volume $\Omega$ follows a certain distribution $P(M)$ which we suppose to be independent of the particular state chosen, in the limit $V \rightarrow \infty$. We expect $P(M)$ to be unchanged by further averaging over the Edwards ensemble, and to be identical to the probability that a fixed window of volume $\Omega$ contains $M$ spheres. Provided the window is much larger than any correlation length for the system, we can use the extensivity of the complexity to approximate the probabilities by

$$
P(M) \propto e^{\Sigma_{N-M, V-\Omega}+\Sigma_{M, \Omega}},
$$

where the terms in the exponent are given by expressions similar to (6). Expanding $\Sigma_{N-M, V-\Omega}$ to first order in $M$ then gives

$$
P(M) \propto e^{\Sigma_{M, \Omega}+\mu M} .
$$

Thus we are led to consider the partition function

$$
Z_{M, \Omega}=\sum_{M} e^{\Sigma_{M, \Omega}+\mu M}
$$

The steepest-descents condition for this sum recovers the expected $\mu=-\partial \Sigma_{M, \Omega} / \partial M$, so that by writing $\Sigma_{M, \Omega}=$ $M \Sigma(\rho)$, with $\rho=M / \Omega$, we obtain

$$
\mu=-d[\rho \Sigma(\rho)] / d \rho .
$$

In the ensemble defined by (12), the variance of $M$ is given by var $M=(\partial M / \partial \mu)_{\Omega}$. By using this in (9) and setting $M=\rho \Omega$ we obtain

$$
S(\mathbf{k} \rightarrow \mathbf{0})=\frac{1}{M}\left(\frac{\partial M}{\partial \mu}\right)_{\Omega}=\frac{1}{\rho} \frac{d \rho}{d \mu} .
$$

The result anticipated in Eq. (5) follows from Eqs. (13) and (14). We note that, from Eqs. (8) and (13), the right-hand side of (14) can also be expressed as $d \rho / d f \equiv \kappa$, a quantity which we call the "compressibility", by a loose analogy with Eq. (1).

We discuss briefly the nature of the averaging procedure. In our treatment we have, for simplicity, considered only Edwards averages over all the possible jammed states. In many 
studies, jammed states are produced by a quench from a fluid state at lower densities and such quenches do not normally result in equal probabilities for all of the states at a given packing fraction. However, the argument leading to Eq. (5) should still apply, provided the complexity remains extensive and the complexity and structure factor are calculated using the same ensemble of states.

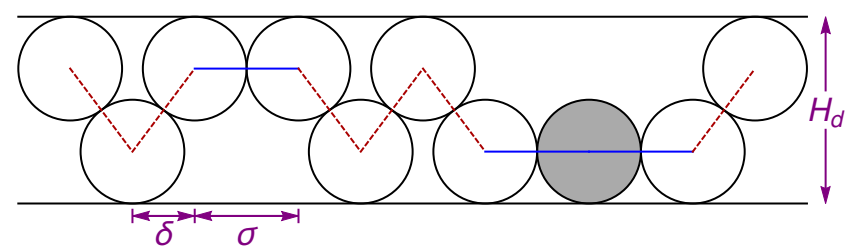

FIG. 1. Local packing arrangements of hard disks of diameter $\sigma$ in a channel of width $H_{d}$. Every disk is in contact with the wall and with two neighboring disks. The shaded disk is not jammed. Red dashed lines ("bonds") join the centers of neighboring disks that are in contact with opposite walls and blue lines join the centers of disks that are in contact with the same wall; the latter can be regarded as "defects" in the zigzag order. The bonds have projections $\delta$ and $\sigma$ along the channel.

We next describe a situation where one can calculate exactly both $S(\mathbf{k} \rightarrow \mathbf{0})$ and also $\Sigma(\rho)$ for a set of jammed states and show that they are indeed related by Eq. (5). These are the jammed states in the system of disks in a narrow channel depicted in Fig. 1. The channel is of width $H_{d}$ and the disks have diameter $\sigma$. It is conventional to express densities via the dimensionless packing fraction $\phi=\pi \rho \sigma^{2} / 4 H_{d}$, where here $\rho=N / L$. Provided $H_{d} / \sigma<1+\sqrt{3 / 4}$, the disks can touch only their nearest neighbors and the sides of the channel. The jammed states of this system are strictly jammed saturated infinite packings. Their only departure from the states envisaged in the Torquato-Stillinger conjecture is that they are not packings in the space $\mathbb{R}^{d}$. Rattlers and voids large enough to accommodate a disk only become possible for wider channels with $H_{d} / \sigma \geq 1+\sqrt{3 / 4}$. Locally, only a small number of configuration types are possible: neighboring disks may touch opposite walls or they may touch the same wall; we refer to the latter as a "defect" in the zigzag arrangement of the disks. The distance between disk centers for two disks touching the same wall is $\sigma$, while for neighbors that touch opposite walls, the separation of their centers, measured along the channel, is $\delta=\sqrt{\sigma^{2}-\left(H_{d}-\sigma\right)^{2}}$. Three successive disks cannot touch the wall to form a jammed state, as the central disk is only locally jammed and may escape by crossing the channel. The Edwards complexity (that is, the total number of jammed states at density $\rho$ ) of this system has been determined by Ashwin and coworkers [7, 8] by two methods, one based on use of the transfer matrix and the other based on a combinatoric approach [7-9].

Let $N_{D}$ be the number of nearest-neighbor pairs of disks that touch the same wall of the channel: these pairs are the defects in the zigzag order [9]. In terms of the concentration of defects, $\theta \equiv N_{D} / N$, the density of the jammed state is

$$
\rho=1 /[\theta \sigma+(1-\theta) \delta]
$$

From the combinatorial approach, the complexity of the state (related to the number of ways it is possible to place the $N_{D}$ defects in the system of $N$ disks $[9,10])$ is

$$
\Sigma=(1-\theta) \ln (1-\theta)-\theta \ln \theta-(1-2 \theta) \ln (1-2 \theta) .
$$

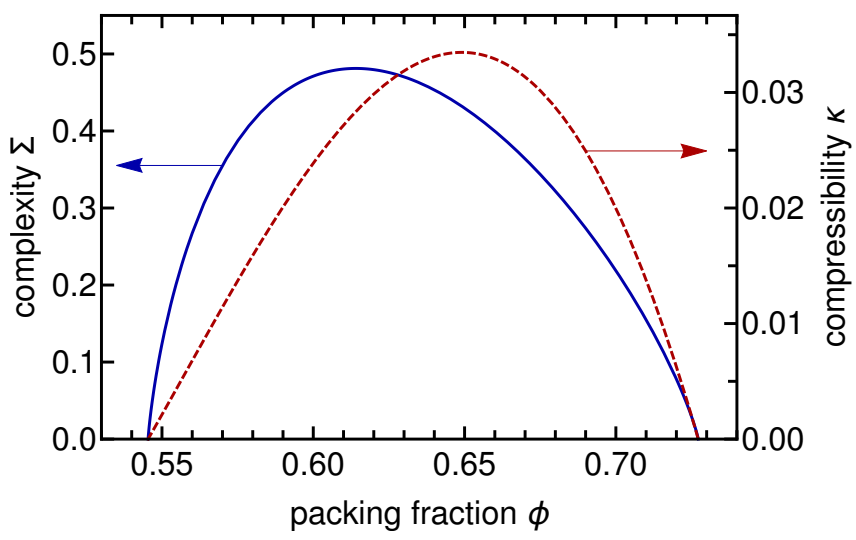

FIG. 2. Complexity per particle $\Sigma$ versus packing fraction $\phi$ for the case $\delta / \sigma=3 / 5$. Also shown is the "compressibility" $\kappa \equiv d \rho / d f$; this quantity is equal to $S(k \rightarrow 0)$ and is nonzero for all $\phi_{\min }<$ $\phi<\phi_{\text {max }}$, implying that the jammed states at these densities are not hyperuniform.

It is then a simple matter to use Eqs. (15) and (16) to calculate the complexity $\Sigma$ as a function of the density $\rho$ or of the packing fraction $\phi$. Figure 2 shows the complexity as a function of the packing fraction for the case $\delta / \sigma=3 / 5$. In Ref. [8] it was argued that if the jammed states are produced by quenching from an initial fluid state, then only the jammed states to the right of the peak in $\Sigma(\phi)$ will be found. Also in Fig. 2 is plotted the right-hand side of Eq. (5). Notice that it only goes to zero, indicating the presence of perfect hyperuniformity, at two exceptional packing fractions, $\phi_{\min }$ and $\phi_{\max }$; the corresponding densities are $\rho_{\min }=2 /(\sigma+\delta)$ and $\rho_{\max }=1 / \delta$. The case of $\phi_{\max }$ arises when no defects are present, i.e. for $\theta=0$. In this case the jammed state has crystalline order and the system is hyperuniform. On the other hand $\phi_{\min }$ arises when $\theta=1 / 2$. This jammed state corresponds to a loosely-packed crystalline structure.

As the starting point for the calculation of the structure factor, we first used the transfer matrix method to obtain the partition function in the $(N, f)$ ensemble, Eq. (7). In (7), the volume function $V\left[C_{N}\right]$ becomes the total length of a configuration of disks, $L=\sum_{i=1}^{N-1} \sigma_{i, i+1}$, where $\sigma_{i, i+1}=\sigma$ for neighboring disks, numbered $i$ and $i+1$, that form a defect and $\sigma_{i, i+1}=\delta$ otherwise. An explicit form for the resulting transfer matrix has been given in Ref. [8]. Let the largest eigenvalue of the transfer matrix be $\lambda_{1}(f)$, so that $Z_{N, f} \approx \lambda_{1}^{N}$ for large $N$. Then $\Sigma_{N, L}-f L=N \ln \lambda_{1}$. As in Eq. (8), $f$ can be 
related to $\rho$ by the steepest descent condition $f=\partial \Sigma_{N, L} / \partial L$. Intuitively, $f$ can be understood as a force that is applied by a piston at the end of the channel; $f$ can be varied to select jammed states of a particular length $L$.

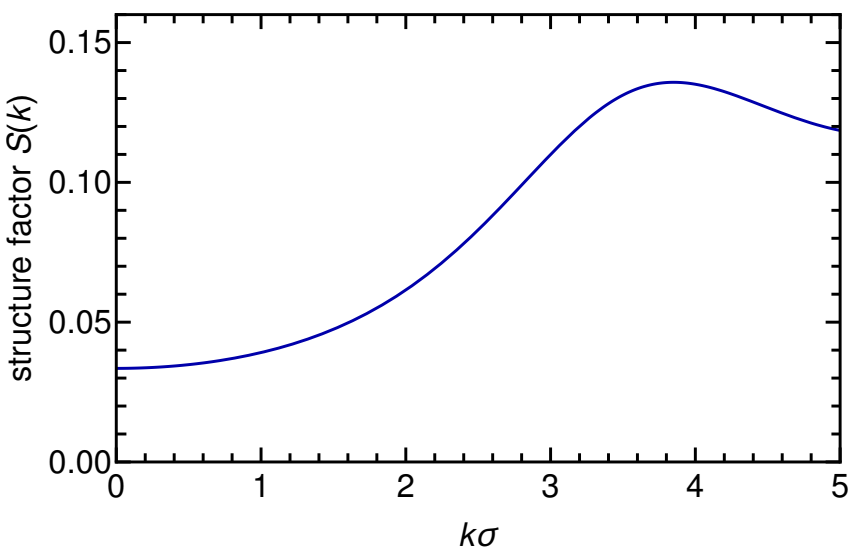

FIG. 3. Structure factor $S(k)$ versus wavenumber $k$ for $\delta / \sigma=3 / 5$ at a packing fraction $\phi=0.649$, which corresponds to the maximum in the compressibility shown in Fig. 2. For small $k, S(k) \rightarrow$ const. + $\mathcal{O}\left(k^{2}\right)$.

The structure factor $S(\mathbf{k})$ can be calculated in closed form from the transfer-matrix solution by the method explained (for a thermal problem) in Ref. [11]. We will consider only the case where $\mathbf{k}$ is in the direction of the channel, as the main interest is the form of $S(k)$ for $k \rightarrow 0$. Figure 3 shows $S(k)$ for small values of $k$. It can be shown analytically to be of the form $S(k)=$ const. $+\mathcal{O}\left(k^{2}\right)$, where the constant is exactly that which would have been obtained from the complexity via Eq. (5). Expressed in terms of $\rho$, the small- $k$ limit of $S(k)$ is given by

$$
S(k \rightarrow 0)=2 \frac{(\rho \sigma-1)\left(1-\rho / \rho_{\max }\right)\left(\rho / \rho_{\min }-1\right)}{\rho(\sigma-\delta)},
$$

which vanishes for $\rho=\rho_{\min }$ and $\rho_{\max }$, corresponding to $\phi=$ $\phi_{\min }$ and $\phi_{\max }$ in Fig. 2.

The results shown in Fig. 3 were calculated for packing fraction $\phi=0.649$ with the ratio $r=\delta / \sigma=3 / 5$. Note that if $r=p / q$ with $p$ and $q$ coprime integers, $S(k)$ will have $\delta$-function peaks for $k=2 \pi m q / \sigma$, where $m \neq 0$ is an integer. The first of these is at $k=10 \pi / \sigma$, which lies outside the range plotted in Fig. 3.

A quite different situation arises if the total complexity is not extensive. An example of such a case is the Fibonacci quasicrystal [12-14], which can be realized as a particular jammed state of our system of disks in a narrow channel system. Let $A$ denote a diagonal (red) bond between neighboring disks and $B$ a horizontal (blue) bond in Fig. 1. Thus the configuration shown in Fig. 1 is $A A B A A A B B A$. The Fibonacci quasicrystal is grown according to the following deterministic dynamical rule [12]

$$
A \rightarrow A B ; \quad B \rightarrow A .
$$

This generates the sequences

$$
A B \rightarrow A B A \rightarrow A B A A B \rightarrow A B A A B A B A \rightarrow \cdots,
$$

in which the numbers of $A$ and $B$ bonds in each block are consecutive members of the Fibonacci sequence 1, 1, 2, 3, $5,8, \ldots$ The dynamical rules do not allow the generation of the configuration in Fig. 1, which contains a $B B$ sequence and is not jammed. For the limit when the final block is infinitely long, the numbers of $A$ and $B$ are such that $N_{A} / N_{B} \equiv(1-\theta) / \theta=(1+\sqrt{5}) / 2$, so that $\theta=(3-\sqrt{5}) / 2$. This value of $\theta$ corresponds to a point to the left of the maximum of the complexity versus $\phi$ plot in Fig. 2, for which the maximum corresponds to $\theta=1 / 2-\sqrt{5} / 10$ [7]. The structure factor of the infinite sequence has been studied in Ref. [13] and is that of a quasicrystal. One can construct windows of length $R$ within the sequence and average over these windows: the number of distinct windows increases as $\sim \exp \left(\Sigma_{R}\right)$. (This connection between the complexity $\Sigma_{R}$ and the number of distinct windows has been studied in some detail by Kurchan and Levine [15].) The complexity $\Sigma_{R}$ is not extensive: for a window of length $R$ in the infinite sequence, the number of distinct windows does not exceed $R$ [12], so $\Sigma_{R}<\ln R$. It can also be shown that the system is hyperuniform [14]: the variance in the number of disks $M$ in a window of length $R$ grows no faster than $\ln R$, so the ratio $\operatorname{var} M /\langle M\rangle \rightarrow 0$ for $R \rightarrow \infty$, implying that the Fibonacci quasicrystal is hyperuniform.

In conclusion, we have shown that hyperuniformity will not arise in a jammed state of hard spheres if the complexity is extensive. The $\mathbf{k} \rightarrow \mathbf{0}$ limit of $S(\mathbf{k})$ is related to the complexity by Eq. (5). We have illustrated this for a simple system for which both the complexity and structure factor could be determined exactly. If the jammed state is generated by dynamical rules that generate a non-extensive complexity, as in our Fibonacci example, then hyperuniformity can arise.

We should like to thank Ludovic Berthier and Salvatore Torquato for helpful correspondence.

[1] S. Torquato, "Hyperuniform states of matter," Phys. Rep. 745, 1 (2018).

[2] Salvatore Torquato and Frank H. Stillinger, "Local density fluctuations, hyperuniformity, and order metrics," Phys. Rev. E 68, 041113 (2003).

[3] Atsushi Ikeda and Ludovic Berthier, "Thermal fluctuations, mechanical response, and hyperuniformity in jammed solids," Phys. Rev. E 92, 012309 (2015).

[4] Atsushi Ikeda, Ludovic Berthier, and Giorgio Parisi, "Largescale structure of randomly jammed spheres," Phys. Rev. E 95, 052125 (2017).

[5] Stefano Martiniani, K. Julian Schrenk, Jacob D. Stevenson, David J. Wales, and Daan Frenkel, "Turning intractable counting into sampling: Computing the configurational entropy of three-dimensional jammed packings," Phys. Rev. E 93, 012906 (2016).

[6] S.F. Edwards and R.B.S. Oakeshott, "Theory of powders," 
Physica A: Statistical Mechanics and its Applications 157, 1080 (1989).

[7] Richard K. Bowles and S. S. Ashwin, "Edwards entropy and compactivity in a model of granular matter," Phys. Rev. E 83, 031302 (2011).

[8] Mahdi Zaeifi Yamchi, S. S. Ashwin, and Richard K. Bowles, "Inherent structures, fragility, and jamming: Insights from quasi-one-dimensional hard disks," Phys. Rev. E 91, 022301 (2015).

[9] M. J. Godfrey and M. A. Moore, "Static and dynamical properties of a hard-disk fluid confined to a narrow channel," Phys. Rev. E 89, 032111 (2014).

[10] P. L. Krapivsky, "Dynamics of repulsion processes," Journal of Statistical Mechanics: Theory and Experiment 2013, P06012 (2013).
[11] J. F. Robinson, M. J. Godfrey, and M. A. Moore, "Glasslike behavior of a hard-disk fluid confined to a narrow channel," Phys. Rev. E 93, 032101 (2016).

[12] Jian Ping Lu, Takashi Odagaki, and Joseph L. Birman, "Properties of one-dimensional quasilattices," Phys. Rev. B 33, 4809 (1986).

[13] Pawel Buczek, Lorenzo Sadun, and Janusz Wolny, "Periodic diffraction pattern for 1d crystals," Acta Phys. Pol. B 36, 919 (2005).

[14] Erdal C. Oğuz, Joshua E. S. Socolar, Paul J. Steinhardt, and Salvatore Torquato, "Hyperuniformity of quasicrystals," Phys. Rev. B 95, 054119 (2017).

[15] Jorge Kurchan and Dov Levine, "Order in glassy systems," Journal of Physics A: Mathematical and Theoretical 44, 035001 (2011). 